\title{
Factores de Riesgo Psicosocial y Satisfacción Laboral en una Empresa Chilena del Área de la Minería
}

\section{PSYCHOSOCIAL RISK FACTORS AND JOB SATISFACTION IN A CHILEAN COMPANY MINING AREA}

\author{
Paola Gómez Rojas', Julia Hernández Guerrero', Maria Doris Méndez Campos² \\ 1. Psicóloga, Universidad de Talca. \\ 2. Psicóloga, Investigador Programa de Investigación en Calidad de Vida y Ambientes Saludables, Facultad de Psicología, Universidad de Talca.
}

\begin{abstract}
RESUMEN
El objetivo de esta investigación es identificar la relación entre Factores de Riesgo Psicosocial y Satisfacción Laboral en trabajadores de una empresa subcontratista del sector minero en Chile. Se utilizó el cuestionario SUSESO-ISTAS 21, adaptación a la población chilena y el Cuestionario de Satisfacción Laboral S20/23 (Meliá y Peiró, 1989), aplicados a una muestra de 100 trabajadores. Los resultados dan cuenta de que existe una relación significativa y negativa entre factores de riesgo y satisfacción laboral; en las dimensiones trabajo activo y posibilidades del desarrollo, apoyo social en la empresa y calidad de liderazgo, compensaciones y doble presencia, indicando que a mayor riesgo psicosocial percibido menor es la satisfacción laboral. Para la dimensión trabajo activo y posibilidades de desarrollo, se encontró que operarios/ayudantes poseen un rango de exposición alto y los demás en un rango medio, además de una relación significativa entre la dimensión exigencias psicológicas según la sección donde trabajan.

Se discuten las implican del estudio para futuras investigaciones e intervenciones organizacionales.
\end{abstract}

(Gómez P, Hernández J, Méndez M, 2014. Factores de Riesgo Psicosocial y Satisfacción Laboral en una Empresa Chilena del Área de la Minería. Cienc Trab. Ene-Abr; 16 [49]: 9-16).

Palabras clave: FACTORES DE RIESGO PSICOSOCIAL, SATISFACCIÓN LABORAL, SUSESO-ISTAS 21, MINERÍA.

\section{ABSTRACT}

The objective of this research is to identify wether a relationship exists between Psychosocial Risk factors and Job satisfaction in a subcontractor workers in the mining sector in Chile. Questionnaire was used SUSESO-ISTAS 21, adapted to the Chilean population and the Job Satisfaction Questionnaire S20/23 (Meliá \& Peiró, 1989), applied to a sample of 100 workers. The results show that there is a significant negative correlation between risk factors and job satisfaction in active labor dimensions and possibilities of development, social support and quality in the company leadership, compensation and dual presence, indicating that greater psychosocial risk lower perceived job satisfaction. For the dimension active work and development opportunities, it was found that operators/attendants have a high exposure range and the other on a mid-range, others in a meaningful relationship between the psychological demands dimension according to the section where they work.

We discuss the study's implications to future research and organizational interventions.

Key words: PSYCHOSOCIAL RISK FACTORS, JOB SATISFACTION, SUSESO-ISTAS 21, MINING.

\section{INTRODUCCIÓN}

Los cambios en la relación empresa trabajador han llevado a que sea necesario considerar todos los factores que conforman el mundo laboral dentro de la organización; en los estados modernos se incluye, entre las responsabilidades de las organizaciones, velar por la seguridad, salud e higiene en el trabajo, lo que implica

Correspondencia / Correspondence:

Ps. Mg. María Doris Méndez

Facultad de Psicologia, Universidad de Talca,

Campus Lircay s/n, Talca. Casilla No. 747

Talca, Chile

Tel.: (5671)201664 • Fax: (5671)201510

e-mail: dmendez@utalca.cl

Recibido: 28 de Diciembre 2013 / Aceptado: 01 de Marzo 2014.

trabajar con seguridad y sin riesgos. Sin embargo, las cifras que reflejan los accidentes laborales, las bajas por enfermedad laboral $\mathrm{y}$ las incapacidades laborales indican que existen situaciones y condiciones en las que la seguridad del trabajo y la promoción de la salud es más una aspiración que una realidad. ${ }^{1}$

Las organizaciones, para poder sobrevivir y prosperar en un contexto dinámico donde el cambio económico y social son continuos e incluso donde la crisis económica entra en juego, necesitan tener empleados motivados y sanos, tanto física como psicológicamente; para conseguirlo, las políticas de recursos humanos deben ser consecuentes con estos valores y con un contexto tan complejo. La evolución de la actividad laboral ha traído consigo una mejora en la calidad de vida de los trabajadores, pero además es responsable de una serie de efectos negativos en la salud de estos. ${ }^{2}$ Acorde a todo lo anterior, las organizaciones modernas están cambiando en una dirección que se basa cada vez más en el conocimiento psicológico, la experiencia y el talento, así como en la auto-gestión y la atención a necesidades individuales y colectivas 
de los empleados, de la organización y de la sociedad en general. $^{3}$

Uno de los cambios más significativos en relación a la comprensión del trabajo es la consideración de la concepción biopsicosocial de la salud y de la necesidad de que ésta se promueva en los lugares de trabajo. ${ }^{4}$

Existe un creciente interés en los denominados factores de riesgos psicosociales, a los que están expuestos los trabajadores en el transcurso de su jornada laboral y que tienen su origen en el terreno de la organización del trabajo y, aunque sus consecuencias no son tan evidentes como los accidentes de trabajo y enfermedades profesionales, no por ello son menos importantes pues se relacionan con aumento de ausentismo, estrés, ansiedad y otros problemas tanto para la organización como para los trabajadores. $^{5}$

Por factores de riesgo psicosocial se entiende: "aquellas condiciones que se encuentran presentes en una situación laboral y que están directamente relacionadas con la organización, el contenido del trabajo, y la realización de la tarea". ${ }^{6}$

Para la Organización Internacional del Trabajo ${ }^{7}$, los factores psicosociales de riesgo son condiciones que conducen al estrés laboral y a otros problemas relativos a la salud y a la seguridad laboral, que comprenden aspectos de trabajo y del entorno del trabajo, como el clima o cultura de la organización, las funciones laborales, las relaciones interpersonales en el trabajo y el diseño y contenido de las tareas (por ejemplo su variedad, significado, alcance, carácter repetitivo, etc.).

Cuenca $^{8}$ representa esta definición gráficamente de la siguiente manera:

Figura 1.

Modelo de Factores Psicosociales en el Trabajo, elaborado por Cuenca, 1996.

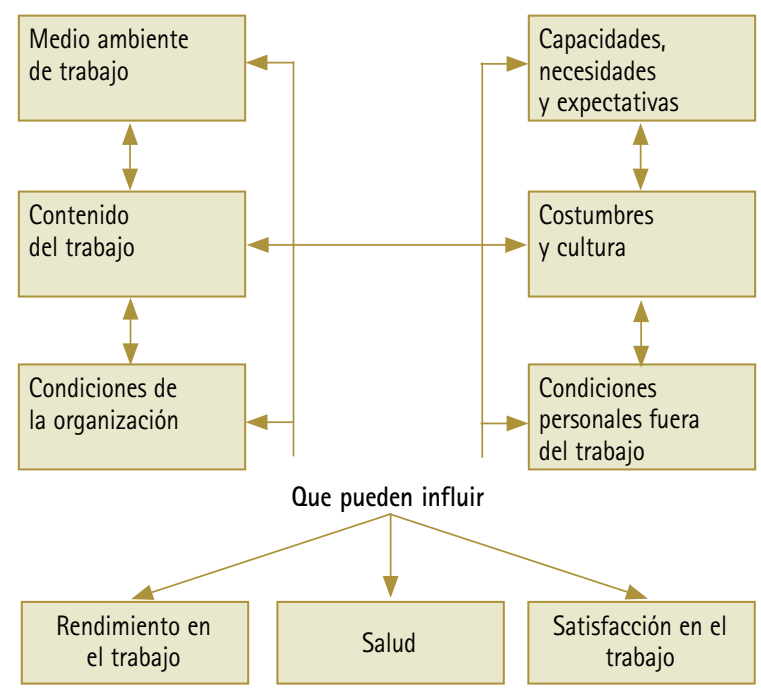

A pesar de haber diversas definiciones, la mayoría de los autores muestra un consenso en los elementos que integran los factores de riesgo psicosocial, como son, por un lado, la interacción entre condiciones de trabajo, necesidades y capacidades del trabajador y, por otro lado, el potencial dañino tanto para la salud de los trabajadores y como para el desarrollo de su trabajo. ${ }^{8}$

La identificación, valoración y control de los factores de riesgo de origen psicosocial y la planificación de las medidas preventivas correspondientes, conduce a una gestión eficaz de las personas en la organización, en el sentido de una mejor adecuación a la tarea a desempeñar, al entorno, a la empresa, aportando en la eficacia de la organización, logrando mejor rendimiento, menor ausentismo y mayor satisfacción. ${ }^{9}$

Por lo tanto, los factores psicosociales de riesgo pueden impactar en forma negativa no sólo a los trabajadores, sino también al funcionamiento de la organización, los trabajadores expuestos a este tipo de factores reducen su desempeño, pudiendo comprometer aspectos como la productividad y la imagen de la empresa, entre otros. ${ }^{10}$

Uno de los mayores problemas actuales derivado de los factores de tipo psicosocial es el estrés. ${ }^{11}$ Son diversas las investigaciones que han definido, que mientras mayor sea la cantidad de roles que la persona debe desempeñar, mayor será la probabilidad de que sufra estrés, por lo cual afectaría en mayor número a las mujeres, debido a la sobrecarga de roles. La calidad de los roles parece ser un moderador del estrés ocupacional en las carreras profesionales de las mujeres, roles de alta calidad mejoran la salud y amortiguan el estrés. ${ }^{12}$ Así como, ser soltero, tener estudios superiores o inferiores al puesto, y estar expuesto a factores psicosociales negativos de tipo laboral, son elementos de riesgo para el desarrollo de estrés y burnout. ${ }^{13}$

Como se señaló, los factores de riesgo psicosocial influyen negativamente en el funcionamiento de las organizaciones en los trabajadores, de ahí la importancia de controlar y gestionar este tipo de riesgos. Una gestión deficiente puede significar altos costos, directos o indirectos para la empresa a consecuencia del aumento del ausentismo, incremento de desvinculaciones voluntarias, baja en la motivación, deterioro de las relaciones laborales y del clima organizacional, amenazas reales de sanciones tanto de tipo civil como administrativas y la consecuente pérdida de reputación que suponen las denuncias y difusión mediática, entre otros. ${ }^{10}$

Según Villalobos ${ }^{14}$, los efectos de los factores de riesgo psicosocial en la salud son diversos y dependen tanto de las caracteristicas de las personas, apreciación de la situación y de los mecanismos de resistencia, así como de las características propias del factor de riesgo. Es así como se pueden identificar dos tipos de respuestas ante los factores de riesgo psicosociales: las respuestas de acomodación pasiva, que conducen a una reducción del interés por participar $\mathrm{y}$ cambiar la realidad, sumiendo al individuo en un ciclo de empobrecimiento personal que limita su capacidad de lucha; - las respuestas de estrés, que tienen componentes subjetivos, fisiológicos $\mathrm{y}$ motores.

En este sentido, Levi $^{7}$ sostiene que como efecto a los factores psicosociales de riesgo y según los tipos de respuesta mencionados en el párrafo anterior, se pueden ocasionar en el individuo enfermedades, dolencias o alteraciones, tanto en el plano físico (cefaleas, migrañas, dolores lumbares, fatiga crónica, trastornos digestivos, hipertensión, alteraciones del sueño, etc.), como psicológico (frustración, culpa, irritabilidad, trastornos cognitivos y de la conducta, depresión, agresividad, neurosis, estrés postraumático en caso de violencia e incluso suicidio).

Por su parte, Moncada, Llorens, Navarro y Kristensen ${ }^{15}$ consideran la relación existente entre los factores psicosociales y la salud, indicando que los efectos de la organización del trabajo se manifiestan a través de diversos mecanismos emocionales, cognitivos, conductuales y fisiológicos.

Otra gran consecuencia de la presencia de factores de riesgos psicosociales son los accidentes de trabajo, generados a través de la presencia de estrés en el trabajo, falta de formación, el exceso de trabajo y tiempo limitado para su ejecución e insatisfacción 
laboral, y la presencia de condiciones físicas de trabajo inadecuadas. $^{9}$

Por otra parte, en los últimos años, diversos estudios muestran el efecto negativo sobre la salud de la falta de recompensas y compensaciones en el trabajo. ${ }^{16}$ Es así como Siegrist et $\mathrm{al}^{7}$ diseñaron y probaron un modelo similar al de demandas-control, cuyas dimensiones esenciales son el "esfuerzo" y la "recompensa social". Siendo las recompensas de tres tipos: económicas a través del salario, la estima (reconocimiento, respeto) y el control del estatus (estabilidad y posibilidad de promoción). ${ }^{17}$ El modelo asegura que el mayor riesgo para la salud lo constituye la combinación de alto esfuerzo (extrínseco: representado por altas demandas o condiciones físicas de trabajo deficientes; o intrínsecos: representado por las formas de afrontamiento o altas necesidades personales de control) y bajo refuerzo (estatus laboral bajo, inseguridad laboral y pocas posibilidades de promoción y desarrollo). ${ }^{18}$

En un estudio realizado en una muestra de trabajadores industriales se probó que un alto esfuerzo combinado con ausencia de recompensas permite predecir un aumento del riesgo de infarto al miocardio que es independiente de los factores de riesgo biomédico. $^{7}$

La integración de estos modelos (demanda - control - apoyo social y esfuerzo recompensa) ha permitido identificar factores de riesgo psicosocial en el trabajo que se relaciona con el estado de salud de los trabajadores, y han servido de fundamento para el desarrollo de instrumentos que permiten la evaluación de estos. ${ }^{19}$

Otro estudio que ha abordado la temática de los factores psicosociales de riesgo es el de Juárez ${ }^{20}$, que identificó los principales factores psicosociales, estrés percibido, estilos de afrontamiento y problemas de salud informados por trabajadores de distintas ocupaciones, tales como administrativos, docentes obreros y enfermeras, dando cuenta de factores psicosociales frecuentes y especificos en cada grupo ocupacional y otros presentes en todos los grupos ocupacionales. El grupo de las enfermeras fue el que reportó mayores problemas de salud y estrés, encontrando también que el apoyo social en el trabajo es una variable relevante. Ansoleaga y Toro $^{21}$ llevaron a cabo un estudio sobre factores psicosociales laborales asociados a riesgo de sintomatología depresiva en trabajadores de una empresa minera, encontrando como resultados la existencia de diferencias en el riesgo de sufrir sintomatología depresiva entre quienes tienen bajo soporte social comparado con quienes tienen alto soporte, alto desbalance esfuerzo-recompensa comparado con quienes tienen bajo desbalance, alta demanda psicológica en comparación con quienes tienen baja demanda, estrés elevado comparado con quienes presentan un bajo nivel de estrés y los que consumen más de un psicotrópico comparado con quienes no consumen. Vera, Sepúlveda y Contreras $^{22}$, estudiaron la relación entre el autorreporte de síntomas físicos y sus correlatos sociodemográficos y psicosociales en trabajadores de la gran minería del cobre, se aplicó un conjunto de instrumentos validados en una muestra de 120 trabajadores varones, en la que los resultados dan cuenta que los trabajadores de menor edad son los que presentan una mayor cantidad de síntomas, insatisfacción con la carga de trabajo, insatisfacción laboral y mayor estrés percibido.

Respecto a la satisfacción laboral existen dos perspectivas en relación al concepto; primero, autores que la conciben como un estado emocional, una actitud o una respuesta afectiva frente al trabajo $\mathrm{y}$, segundo, autores que conciben la satisfacción laboral como una comparación entre expectativas, exigencias y beneficios que el puesto ofrece. ${ }^{23}$ Desde el primer enfoque está Locke ${ }^{24}$ que la define como "un estado emocional positivo, o agradable derivado de la valoración que el individuo hace del resultado de su trabajo o sus experiencia con el mismo", y Bravo et al. ${ }^{25}$ que entienden la satisfacción laboral como una actitud o conjunto de actitudes desarrollados por la persona hacia la situación de trabajo. ${ }^{26}$

En cuanto a las definiciones enmarcadas en la segunda perspectiva tenemos las propuestas por Mumford, Holland y Michalos ${ }^{26}$, quienes la consideran como el nivel de ajuste que el sujeto experimenta entre sus necesidades, sus expectativas y las prestaciones que otorga la organización.

También existen definiciones que integran ambas perspectivas, como es la de Gibson et al. ${ }^{27}$, que proponen que la satisfacción laboral es una predisposición que los sujetos proyectan acerca de sus funciones laborales y la definen como "la actitud resultado de sus percepciones sobre el trabajo, basadas en factores relativos al ambiente en que se desarrolla el mismo, como es el estilo de dirección, las políticas y procedimientos, la satisfacción de los grupos de trabajo, las condiciones laborales y el margen de beneficios".

Según Pérez ${ }^{28}$, estas características son las que configurarán los umbrales personales de satisfacción o insatisfacción, tal como se puede ver en la Figura 2.

Figura 2.

Variables que inciden en la satisfacción laboral, extraído de Pérez, 2006.

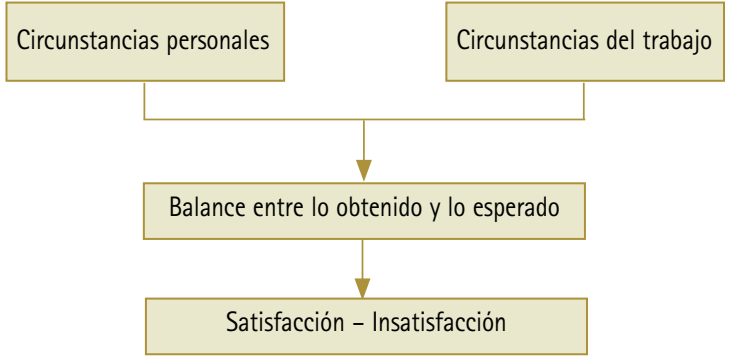

Asimismo, es importante destacar que la satisfacción laboral puede ser un sentimiento de bienestar o actitud generalizado o global, abarcando todos los aspectos o dimensiones del trabajo, por lo que se puede hablar de "satisfacción laboral general", o este sentimiento de satisfacción o bienestar puede estar referido a alguno de los aspectos o dimensiones del trabajo, por lo que se puede hablar entonces de "satisfacción laboral por dimensiones". ${ }^{29}$ Lo anterior implica un modelo compensatorio, de modo que un elevado nivel de satisfacción en una determinada dimensión de trabajo puede compensar deficiencias existentes en otras determinadas esferas del trabajo, de modo que unas dimensiones pueden ser compensadas y condicionadas por otras. ${ }^{29}$

Muchas de las investigaciones sobre satisfacción laboral, en el extranjero como en Chile, tienen relación con profesionales de la salud, ya que el aumento de la satisfacción del profesional incide directamente con la calidad del servicio al optimizar la relación con el paciente y aumentar la calidad de sus cuidados. ${ }^{30}$

Fernández y Paravic ${ }^{31}$ comparan los niveles de satisfacción laboral en enfermeras de centros hospitalarios públicos y privados, donde las del sector privado presentan un mayor nivel de satisfacción que aquellas del servicio público.

Otras investigaciones ${ }^{32,33}$ señalan que la satisfacción laboral es un importante predictor de conductas disfuncionales como el ausentismo, rotación, accidentabilidad, abandono del trabajo. 
Machuca y Vera ${ }^{34}$ afirman que en Chile la investigación psicosocial sobre la relación entre salud y trabajo en poblaciones ocupacionales es escasa, mientras que en el ámbito internacional existe abundante evidencia que da cuenta de esta relación; respecto, como se indicó anteriormente, se ha estudiado la relación entre estrés laboral, enfermedades físicas y desajuste psicológico, la relación entre lugar de trabajo y enfermedades cardiovasculares, entre otros. ${ }^{35,22}$

En Chile, el alza experimentada en los últimos años por licencias médicas referidas a trastornos mentales y del comportamiento ${ }^{36}$ evidencia la importancia de considerar esta asociación como un tema de preocupación, siendo las patologías más asociadas a este aumento la depresión, ansiedad y estrés. ${ }^{21}$

Un estudio acerca de los factores psicosociales que inciden en el estrés laboral muestra que ciertas profesiones, como las relacionadas al trabajo en la minería, en las que existen situaciones laborales objetivas con mucha exigencia contextual, son potencialmente más estresantes que otras. ${ }^{37}$ Asimismo, un estudio europeo expone que los trabajadores más castigados por el estrés son los mineros, albañiles y policías. ${ }^{38}$

Conociendo los datos anteriormente descritos y siendo escasa la investigación a nivel nacional en este tema y en esta población en particular, es que resulta relevante este estudio, a fin de profundizar en el conocimiento de relaciones que puedan existir entre la exposición a factores de riesgo psicosocial y variables como satisfacción laboral, características sociodemográficas y laborales en trabajadores ligados al ámbito de la minería de tipo subterránea. Especialmente, porque este sector productivo es de gran importancia al desarrollo económico del país, además de constituir una actividad que ha llegado a crear su propia cultura y porque, en la medida que se conozcan los elementos psicosociales nocivos para la seguridad y salud se pueden implementar medidas correctivas y preventivas adecuadas al entorno específico del trabajo. ${ }^{21}$

El objetivo del siguiente estudio es identificar si existe relación entre Factores de Riesgo Psicosocial y Satisfacción Laboral en trabajadores de una empresa subcontratista del sector minero en Chile.

Algunas de las hipótesis que se deseaba contrastar eran que existe una relación significativa y negativa entre presencia de factores de riesgo psicosocial y nivel de satisfacción laboral en los trabajadores de la muestra; a mayor presencia del factor de riesgo psicosocial exigencias psicológicas, menor nivel de satisfacción laboral en los trabajadores de la muestra; a menor presencia del factor de riesgo psicosocial trabajo activo y posibilidades de desarrollo, menor satisfacción laboral en los trabajadores de la muestra; a menor presencia del factor de riesgo psicosocial apoyo social en la empresa y calidad de liderazgo, menor satisfacción laboral en los trabajadores de la muestra; a menor presencia del factor de riesgo psicosocial compensaciones, menor satisfacción laboral en los trabajadores de la muestra; a mayor presencia del factor de riesgo psicosocial doble presencia, menor satisfacción laboral en los trabajadores de la muestra.

\section{MÉTODO}

El presente estudio es de tipo correlacional, con un diseño no experimental, transversal. La muestra es de tipo no probabilística; por conveniencia estuvo compuesta por 100 trabajadores de una empresa subcontratista del sector minero de la sexta región, un $95,9 \%$ corresponde a hombres y el 4,1\% corresponde a mujeres, la mayoría de ellos se encuentra en un rango de edad de entre 26 y 35 años (35\%), seguido por 36 y 45 años (28\%), luego los menores de 26 años (23\%), entre 46 y 55 años (12\%) y finalmente el menor porcentaje está en los mayores de 55 años con un $2 \%$ de muestra. En cuanto a las secciones en la que los trabajadores realizan sus funciones, el porcentaje mayor corresponde a SAG 1 y $2(37,1 \%)$, seguido por Chancado Secundario $(31,4 \%)$, luego Departamento de Prevención de Riesgos (11,4\%), UPH $(7,1)$, seguido por Administración (5,7\%), Chancado Terciario (4,3\%) y finalmente Recursos Humanos (1,4\%) .

En cuanto al procedimiento, previa aplicación de los instrumentos de medida se remitió una carta de solicitud de estudio al Gerente de la empresa y al jefe de prevención de riesgos de la organización, solicitando la autorización firmada de ellos junto a un representante de los trabajadores, en la cual se especificaba las características de la investigación a realizar y se garantizaba la absoluta confidencialidad en el tratamiento de los datos. Luego se aplicó una carta de consentimiento informado, el cuestionario sociodemográfico y sociolaboral, el cuestionario de factores de riesgo psicosocial SUSESO ISTAS-21 y el cuestionario de satisfacción laboral S20/23.

El SUSESO ISTAS 21 evalúa riesgos psicosociales en el trabajo y se basa conceptualmente en la teoría general del estrés, integrando las dimensiones de los modelos demanda control-apoyo social de Karasek y Therorell, y esfuerzo recompensa (ERI) de Siegrist. ${ }^{19}$ Es el resultado de la adaptación, validación y estandarización del Método ISTAS 21 - COPSOQ en la población trabajadora chilena. ${ }^{39}$ No se tiene información psicométrica respecto del instrumento adaptado en Chile, por esta razón se hizo el análisis de la confiabilidad del instrumento en esta aplicación obteniendo un alfa de 0,92, lo cual se considera adecuado. El SUSESO ISTAS 21 contiene 115 itemes, agrupados en 21 subdimensiones psicosociales laborales, agrupadas en 5 grandes dimensiones: 1.Exigencias psicológicas. 2.- Trabajo activo y posibilidades de desarrollo de habilidades. 3.- Apoyo social en la empresa y calidad de liderazgo. 4.- Compensaciones. 5.- Doble presencia. Los resultados de la evaluación se comparan con los valores de referencia poblacionales, y las puntuaciones expresan la media para cada una de las dimensiones (estandarizados de 0 a 100), donde los puntajes se separan en terciles para cada una de las dimensiones y subdimensiones, clasificándolos en rangos "bajo", "medio" y "alto" la exposición a cada uno de los factores de riesgo psicosocial. ${ }^{19}$

Para evaluar los niveles de Satisfacción Laboral de la muestra se utilizó el cuestionario de Satisfacción Laboral S20/23 de J. L. Meliá y J. M. Peiró. ${ }^{32}$ El instrumento presenta un valor de alfa que oscila entre 0,76 y 0,89 . El cuestionario está compuesto por 23 itemes tipo likert que van de 1 a 5 , contempla 5 factores medibles que permiten evaluar agrupadamente la satisfacción con respecto a 1.- la supervisión, 2.- el ambiente físico laboral, 3.- prestaciones recibidas, 4.- satisfacción intrínseca del trabajo, 5.- participación.

Se elaboró también una Pauta de preguntas sobre Antecedentes Sociodemográficos y Laborales, que consignó información sobre sexo, edad, estado civil, cargo, sección, antigüedad laboral, tipo de contrato, escolaridad y horario de trabajo. Esta información fue complementada con los datos de este tipo que recoge el instrumento SUSESO-ISTAS 21. 
Tabla 1.

Estadísticos descriptivos y Anova para los factores del Cuestionario de Evaluación de Riesgos Psicosociales en el Trabajo SUSESO-ISTAS 21 en la variable Sección.

\begin{tabular}{|c|c|c|c|c|c|c|c|}
\hline & Sección & $\mathrm{N}$ & Media & $\begin{array}{l}\text { Desviación } \\
\text { típica }\end{array}$ & $\mathrm{gl}$ & $F$ & Sig. \\
\hline \multirow{16}{*}{$\begin{array}{l}\text { Exigencias } \\
\text { Psicológicas }\end{array}$} & Chancado & & & & & & \\
\hline & Primario & 1 & 51,07100 & & 7 & 2,162 & 0,050 \\
\hline & Chancado & & & & & & \\
\hline & Secundario & 22 & 46,52968 & 10,375883 & & & \\
\hline & Chancado & & & & & & \\
\hline & Terciario & 3 & 49,94000 & 13,403371 & & & \\
\hline & Administración & 4 & 45,91475 & 5,049840 & & & \\
\hline & Recursos Humanos & 1 & 22,67800 & & & & \\
\hline & $\begin{array}{l}\text { Departamento } \\
\text { de Prevención } \\
\text { de Riesgos }\end{array}$ & 8 & 55,54638 & 12,660 & & & \\
\hline & SAG 1 y 2 & & & & & & \\
\hline & (Molienda & & & & & & \\
\hline & $\begin{array}{l}\text { convencional } \\
\text { y flotación) }\end{array}$ & 26 & 44,45358 & 14,579 & & & \\
\hline & UPH (Unidad & & & & & & \\
\hline & de proceso & & & & & & \\
\hline & Hidrometalúrgico) & 5 & 32,71380 & 7,701 & & & \\
\hline & Total & 70 & 45,63734 & 12,997 & & & \\
\hline \multirow{15}{*}{$\begin{array}{l}\text { Trabajo activo } \\
\text { y posibilidades } \\
\text { de desarrollo }\end{array}$} & Chancado Primario & 1 & 48,92800 & & 7 & 3,628 & 0,002 \\
\hline & Chancado & & & & & & \\
\hline & Secundario & 22 & 50,44050 & 16,725764 & & & \\
\hline & Chancado Terciario & 3 & 55,81300 & 11,294536 & & & \\
\hline & Administración & 4 & 25,69900 & 11,030564 & & & \\
\hline & Recursos Humanos & 1 & 44,40400 & & & & \\
\hline & Departamento de & & & & & & \\
\hline & Prevención & & & & & & \\
\hline & de Riesgos & 8 & 28,66750 & 8,947451 & & & \\
\hline & SAG 1 y 2 & & & & & & \\
\hline & $\begin{array}{l}\text { (Molienda } \\
\text { convencional }\end{array}$ & & & & & & \\
\hline & y flotación) & 26 & 46,53565 & 13,331835 & & & \\
\hline & $\begin{array}{l}\text { UPH (Unidad } \\
\text { de proceso }\end{array}$ & & & & & & \\
\hline & Hidrometalúrgico) & 5 & 38,20200 & 10,880570 & & & \\
\hline & Total & 70 & 44,33621 & 15,638905 & & & \\
\hline \multirow{14}{*}{$\begin{array}{l}\text { Compensa- } \\
\text { ciones }\end{array}$} & Chancado Primario & 1 & 24,44400 & & 7 & 2,731 & 0,015 \\
\hline & Chancado & & & & & & \\
\hline & Secundario & 22 & 37,92886 & 17,983340 & & & \\
\hline & Chancado Terciario & 3 & 73,51800 & 6,946348 & & & \\
\hline & Administración & 4 & 26,11075 & 20,092573 & & & \\
\hline & Recursos Humanos & 1 & 35,55500 & & & & \\
\hline & Departamento de & & & & & & \\
\hline & $\begin{array}{l}\text { Prevención } \\
\text { de Riesgos }\end{array}$ & 8 & 29,86075 & 13,212977 & & & \\
\hline & SAG 1 y 2 & & & & & & \\
\hline & (Molienda & & & & & & \\
\hline & $\begin{array}{l}\text { convencional } \\
\text { y flotación) }\end{array}$ & 26 & 41,28169 & 16,782237 & & & \\
\hline & $\begin{array}{l}\text { UPH (Unidad } \\
\text { de proceso }\end{array}$ & & & & & & \\
\hline & Hidrometalúrgico) & 5 & 32,55520 & 18,813420 & & & \\
\hline & Total & 70 & 38,49167 & 18,359985 & & & \\
\hline
\end{tabular}

Para el análisis de los datos de este estudio se utilizó el programa estadístico SPSS para Windows versión 15.0, para análisis descriptivos se utilizó la Prueba T y Anova de un factor. Para evaluar la correlación entre factores psicosociales de riesgo laboral y satisfacción laboral se calculó el coeficiente de correlación de Pearson entre cada dimensión factores psicosociales de riesgo y sus escalas, con los puntajes totales del instrumento de satisfacción laboral y cada una de sus dimensiones.

\section{RESULTADOS}

Se encuentran diferencias estadísticamente significativas dentro de los grupos en la dimensión trabajo activo y posibilidades de desarrollo $(\mathrm{F}[3]=8,053, \mathrm{p}<0,05)$ en la variable cargo. Los cargos jefatura/supervisor $($ media $=32,60)$, profesionales $($ media $=29,65)$ y administrativos/técnicos (media $=33,63$ ) se ubican en un rango medio de exposición a este factor y quienes se desempeñan como operarios/ayudantes (media $=47,99$ ) se encuentran en un rango de exposición alto.

En la Tabla 1 se indica que para la variable sección con respecto al factor de riesgo psicosocial exigencias psicológicas $(F[7]=2,162$ $p<0,050$ ), se encontraron diferencias estadisticamente significativas entre las medias de los grupos, por lo que los trabajadores de las secciones; chancado primario (media $=51,07$ ), chancado secundario (media $=46,52$ ), chancado terciario (media $=49,94)$ y departamento de prevención de riesgos (media $=55,54$ ) se encuentran en un rango medio de exposición al factor, mientras que los trabajadores que se desempeñan en administración (media $=45,91$ ), recursos humanos (media $=22,67)$, SAG 1 y $2($ media $=44,45) \mathrm{y}$ UPH (media $=32,71$ ) se encuentran en un rango de baja exposición a este factor, es decir, se encuentran en la situación más benévola para la salud en relación a esta dimensión.

Para la variable sección, respecto al factor trabajo activo y posibilidades de desarrollo, también existen diferencias estadísticamente significativas al comparar las medias de los grupos $(F[7]=3,62 ; p$ $<0,05$ ), por lo tanto se puede observar que los trabajadores que se desempeñan en chancado primario (media $=48,92$ ), chancado secundario (media $=50,44)$, chancado terciario $($ media $=55,81$ ), recursos humanos (media $=44,40)$ y SAG 1 y 2 (media $=46,53$ ) se ubican en un rango de exposición alto a este factor, es decir, están expuestos a la situación más desfavorable para la salud en cuanto a esta dimensión; quienes se desempeñan en el departamento de prevención de riesgos (media $=28,66)$ y UPH (media $=38,20)$ están en un rango medio y los trabajadores de la sección administración (media $=25,69)$ presentan un rango de exposición bajo a este factor, es decir, se encuentran en la situación más benévola para la salud respecto a este factor.

Para el factor compensaciones $(\mathrm{F}[7]=2,731, \mathrm{p}<0,05)$ también se encontraron diferencias estadísticamente significativas entre las medias para la variable sección. Se ubican en un rango de exposición medio a este factor las siguientes secciones: chancado primario $($ media $=24,44)$, chancado secundario $($ media $=37,92)$, administración (media $=25,11$ ), recursos humanos (media $=35,55$ ), departamento de prevención de riesgos (media $=29,86)$, SAG $1 \mathrm{y}$ 2 (media $=41,28)$ y UPH (media $=32,55)$; mientras que los trabajadores que se desempeñan en chancado terciario (media $=73,51$ ) están en un rango de exposición alto a este factor.

Respecto a la variable Satisfacción Laboral y la variable cargo se encontraron diferencias significativas en todas las dimensiones de la escala, indice general de satisfacción laboral $(\mathrm{F}[3]=5,763 ; \mathrm{p}<$ $0,05)$, satisfacción intrínseca del trabajo $(\mathrm{F}[3]=4,407 ; \mathrm{p}<0,05)$, satisfacción con las prestaciones $(\mathrm{F}[3]=7,938$; $\mathrm{p}<0,05)$, satisfacción con el ambiente físico $(\mathrm{F}[3]=4,618 ; \mathrm{p}<0,05)$, satisfacción con la supervisión $(\mathrm{F}[3]=3,095, \mathrm{p}<0,05)$ y satisfacción con la participación $(\mathrm{F}[3]=6,207 ; \mathrm{p}<0,05)$.

Donde el índice general de satisfacción exhibe las puntuaciones más altas en la muestra, puntuando en el nivel "indiferente", es en los trabajadores que ocupan cargos de Jefatura/Supervisor, Profesionales y Administrativos, en contraposición con los traba- 
Tabla 2.

Estadisticos descriptivos y Anova para las dimensiones de la escala de satisfacción laboral en la variable antigüedad laboral.

\begin{tabular}{|c|c|c|c|c|c|c|c|}
\hline & Antigüedad Laboral & I N & Media & $\begin{array}{l}\text { Desviación } \\
\text { típica }\end{array}$ & $\mathrm{gl}$ & $\mathrm{F}$ & Sig. \\
\hline \multirow{5}{*}{$\begin{array}{l}\text { Satisfacción } \\
\text { intrinseca } \\
\text { del trabajo }\end{array}$} & De 0 a 6 meses & 10 & 4,80 & 1,274 & 3 & 3,354 & 0,022 \\
\hline & $\begin{array}{l}\text { Más de } 6 \text { meses } \\
\text { y hasta } 2 \text { años }\end{array}$ & 31 & 4,08 & 1,608 & & & \\
\hline & & 56 & 4,99 & 1,112 & & & \\
\hline & $\begin{array}{l}\text { Más de } 5 \text { años } \\
\text { y hasta } 10 \text { años }\end{array}$ & 3 & 5,00 & 1,000 & & & \\
\hline & Total & 100 & 4,69 & 1,345 & & & \\
\hline \multirow{5}{*}{$\begin{array}{l}\text { Satisfacción } \\
\text { con las } \\
\text { prestaciones }\end{array}$} & De 0 a 6 meses & 10 & 3,82 & 1,318 & 3 & 2,941 & 0,037 \\
\hline & $\begin{array}{l}\text { Más de } 6 \text { meses } \\
\text { y hasta } 2 \text { años }\end{array}$ & 31 & 2,92 & 1,240 & & & \\
\hline & $\begin{array}{l}\text { Más de } 2 \text { años } \\
\text { y hasta } 5 \text { años }\end{array}$ & 56 & 3,80 & 1,476 & & & \\
\hline & $\begin{array}{l}\text { Más de } 5 \text { años } \\
\text { y hasta } 10 \text { años }\end{array}$ & 3 & 4,00 & 1,778 & & & \\
\hline & Total & 100 & 3,53 & 1,439 & & & \\
\hline
\end{tabular}

jadores que se desempeñan en cargos Operativos/Ayudantes, quienes obtienen las puntuaciones más bajas en satisfacción laboral puntuando en el nivel "algo insatisfecho".

Como se observa en la Tabla 2, sólo se encontraron diferencias estadisticamente significativas entre la variable antigüedad laboral y las dimensiones satisfacción intrínseca del trabajo $(\mathrm{F}[3]=3,354$; $\mathrm{p}<$ $0,05)$ y satisfacción con las prestaciones $(F[3]=2,941 ; p<0,05)$.

En la variable sección se encontraron diferencias significativas en las dimensiones satisfacción con el ambiente físico $(\mathrm{F}[7]=6,829 ; \mathrm{p}<$ $0,05)$ y satisfacción con la participación $(F[7]=2,474 ; p<0,05)$, además del índice general de la escala $(\mathrm{F}[7]=4,381 ; \mathrm{p}<0,05)$. En donde el índice de satisfacción general se observa con menores niveles de satisfacción laboral es en los trabajadores de chancado terciario (media $=1,95$ ) quienes muestran un nivel "muy insatisfecho", mientras que los trabajadores de la sección administración (media $=5,08$ ) presentaron puntuaciones superiores categorizando en el nivel "algo satisfechos". En cuanto a la dimensión satisfacción con el ambiente físico los trabajadores de la sección chancado primario (media $=2,00$ ) presentaron los puntajes más bajos, cayendo en el nivel "bastante Insatisfecho", mientras que los que mostraron puntajes superiores fueron los trabajadores de recursos humanos (media $=5,80$ ) y prevención de riesgos (media $=5,88$ ) que categorizan en el nivel "algo satisfecho" y los trabajadores de administración (media $=6,20$ ), quienes se sitúan en un nivel superior "bastante satisfecho". Finalmente, en la dimensión satisfacción con la participación los trabajadores que mostraron puntajes más bajos son los de la sección chancado terciario (media $=3,67$ ) quienes se situaron en el nivel "algo insatisfechos", mientras que en el nivel "bastante satisfecho" se encuentran los trabajadores de administración (media = 6,33 ) y prevención de riesgos (media $=6,08$ ), siendo estos quienes mostraron los puntajes más altos.

En cuanto al análisis de los resultados de la correlación de la satisfacción laboral (evaluado por las 5 escalas del S20/23) con la dimensión trabajo activo y posibilidades de desarrollo, se encontraron correlaciones negativas significativas entre las 5 escalas, que son: satisfacción intrínseca del trabajo $(r=-0,489, p<0,05)$, satisfacción con las prestaciones $(\mathrm{r}=-0,527, \mathrm{p}<0,05)$, satisfacción con el ambiente físico $(r=-0,404, p<0,05)$, satisfacción con la supervisión $(\mathrm{r}=-0,381, \mathrm{p}<0,05)$ y satisfacción con las participación $(\mathrm{r}=-0,459$, $p<0,05)$, incluyendo el incide general de satisfacción $(r=-0,593, p$
$<0,05)$. Por lo tanto, a mayor riesgo psicosocial percibido en el trabajo y posibilidades del desarrollo menor es la satisfacción laboral en los trabajadores en todas las dimensiones de la escala.

El análisis de los resultados de la correlación de Pearson entre apoyo social en la empresa y calidad de liderazgo y satisfacción laboral muestra una correlación negativa y significativa entre estas variables $(\mathrm{r}=-0,661, \mathrm{p}<0,05)$, con un grado de asociación moderado.

De acuerdo al análisis de correlación entre compensaciones y satisfacción laboral, se puede establecer que se da una correlación negativa entre la variable compensación y el índice general de satisfacción laboral; esta correlación es estadísticamente significativa y tiene un grado de asociación bajo $(r=-0,317, \mathrm{p}<0,05)$

Entre doble presencia y satisfacción laboral existe un bajo grado de asociación lineal, negativo y significativo $(r=-0,319, p<0,05)$.

\section{CONCLUSIÓN Y DISCUSIÓN}

Se describió los resultados en las 5 dimensiones del instrumento, donde exigencias psicológicas, apoyo social en la empresa y calidad de liderazgo, compensaciones y doble presencia se encuentran en rango medio de exposición a los factores de riesgo, mientras la dimensión trabajo activo y posibilidades de desarrollo se encuentra en un rango alto de exposición, es decir, los trabajadores en este factor están expuestos a la situación más desfavorable para la salud.

Además, se encontró que existe relación significativa entre la dimensión Exigencias Psicológicas en cuanto a la sección, en la que el personal de Chancado Primario, Chancado Secundario, Chancado Terciario y Departamento de Prevención de Riesgos perciben una exposición media al factor, considerando que deben esconder emociones, evalúan que el nivel de complejidad y variabilidad y/o el tiempo establecido para realizar las tareas no es el más adecuado, al igual que la formación que requieren para realizar la ocupación. Mientras que en la sección de Administración, Recursos Humanos, SAG 1 y 2 y UPH esta percepción es diferente, ya que consideran que los procesos mencionados anteriormente son adecuados. Lo encontrado se condice con el modelo inicial Demanda-Control40, donde la condición de mayor riesgo está dada por altas exigencias psicológicas y bajo control de tarea, como es el caso de los trabajadores de niveles jerárquicos bajos. ${ }^{19}$

Por su parte, la dimensión Trabajo Activo y Posibilidades de Desarrollo obtuvo diferencias estadísticamente significativas en cuanto a las variables cargo y sección. Es así como los trabajadores del cargo Operario/Ayudante consideran que no tienen influencia sobre su trabajo ni en la relación con los compañeros, sienten que su trabajo no es fuente de oportunidades de desarrollo de sus habilidades y conocimientos, sienten que no tienen influencia sobre los tiempos de descanso, como vacaciones o detenciones temporales; no experimentan sentido del trabajo, es decir, no lo pueden relacionar con otros valores o fines aparte de los puramente instrumentales y no se sienten implicados en la empresa.

Asimismo, en relación a la variable sección los trabajadores Chancado Primario, Chancado Secundario, Chancado Terciario, Recursos Humanos, y SAG 1 y 2 comparten la evaluación en rangos altos de exposición al factor de riesgo Trabajo Activo y Posibilidades de Desarrollo, mientras que la sección Administración es la que muestra la condición más favorable para la exposición a este factor.

Lo anteriormente expuesto coincide con lo expuesto por Moreno, Llorens y Moncada ${ }^{16}$, quienes señalan que los trabajadores manuales 
tienen un nivel de control inferior al de trabajadores no manuales; esto se ve reflejado en la muestra pues se pudo establecer que mayoritariamente los trabajadores de los cargos Operario/Ayudante y de las secciones productivas (a excepción del área de recursos humanos) como Chancado Primario, Chancado Secundario, Chancado Terciario, SAG 1 y 2, cuyas tareas son principalmente manuales, identifican mayores riesgos en esta área en comparación con los trabajadores del área administrativa.

En cuanto a la dimensión Compensaciones, se pudo observar diferencias significativas solo a nivel de la sección, siendo los trabajadores de Chancado Terciario quienes no sienten reconocimiento de los superiores y del esfuerzo realizado para desempeñar el trabajo, sintiendo inseguridad con respecto al contrato de trabajo (posibles despidos) e inseguridad en relación a las características mismas del trabajo (por ejemplo, cambios de lugar de trabajo, de turnos, etc.). Esta situación se explica a través del modelo esfuerzo recompensa de Siegrist ${ }^{7}$, que postula que el estrés laboral se produce porque existe una falta de balance (equilibrio) entre el esfuerzo y la recompensa obtenida, generándose desequilibrio a partir de un elevado esfuerzo, un salario inadecuado y un bajo control sobre el propio estatus ocupación, como puede ser el caso de los trabajadores de Chancado. En cuanto a la satisfacción de los trabajadores con su trabajo, se evidenciaron diferencias significativas a nivel de la variable cargo en todas las dimensiones, incluyendo el índice general de satisfacción, en donde los cargos que presentan los mayores niveles de satisfacción laboral son Jefatura/Supervisor, Profesionales y Administrador/ Técnico, mientras que los que mostraron niveles más bajos fueron los cargos Operario/Ayudante. Lo anterior es coincidente con lo planteado por Sánchez y Muchinsky ${ }^{26}$ quienes proponen que los empleados con puestos con mayor estatus poseen niveles superiores de satisfacción que los trabajadores en puestos inferiores, debido a una mayor autonomía y posibilidades de liderazgo del puesto de trabajo.

En relación a la variable sección, se encuentran diferencias estadísticamente significativas en dos de las dimensiones de la escala (satisfacción con el ambiente físico y satisfacción con la participación) y en el Índice General, las que son: Satisfacción con el Ambiente Físico y Satisfacción con la Participación, en donde los trabajadores de las secciones Chancado Terciario, Chancado Secundario, Chancado Terciario y SAG 1 y 2 muestran los niveles más bajos de satisfacción; esto quiere decir que los trabajadores de esta sección se sienten a disgusto con el entorno físico y el espacio en el lugar de trabajo, la limpieza, higiene y salubridad, la temperatura, la ventilación y la iluminación. Lo anterior coincide con lo planteado por Muchinsky ${ }^{26}$ quien dice que todos los aspectos mencionados anteriormente afectan los niveles de satisfacción al ser incómodos y/o peligrosos y al no contar con herramientas e instalaciones adecuadas. Mientras que en relación a la otra dimensión (Satisfacción con la Participación), los trabajadores de Chancado Terciario, Chancado Secundario, Chancado Primario y SAG 1 y 2 están insatisfechos con la participa- ción en las decisiones ya sea a nivel del grupo de trabajo o de las relacionadas con la tarea a realizar. Mientras que los trabajadores de las secciones Administración y Prevención de Riesgos expresan satisfacción en relación a lo mencionado anteriormente. Esto se condice con lo expuesto por Meliá y Peiró ${ }^{32}$ quienes plantean que la satisfacción con la participación viene dada por la capacidad para decidir aspectos relativos con el trabajo, participación en las decisiones relativas al área de trabajo, grupo de trabajo y a la empresa, situación que no se da para las secciones de Chancado Primario, Secundario y Terciario y SAG 1 y 2 presumiblemente por la naturaleza de sus trabajos.

En la relación entre las dos variables del estudio: Factores de Riesgo Psicosocial y Satisfacción Laboral y sus respectivas dimensiones, se pudo observar que existe una correlación negativa significativa entre ellas.

Lo anterior concuerda con las publicaciones encontradas, las que señalan que los factores de riesgo psicosocial influyen en la satisfacción laboral en contextos organizacionales en que las condiciones psicosociales, de seguridad y medio ambiente laboral físico implican riesgo para la salud de los trabajadores, como sería el caso del personal que desarrolla su trabajo en algunas áreas de la minería. ${ }^{8,21}$ Así tambien, el comportamiento de estas variables se pueden explicar a partir de la teoría general del estrés que integra los modelos Demanda-Control-Apoyo Social de Karasek y Theorell ${ }^{41}$ y EsfuerzoRecompensa (ERI) de Siegrist ${ }^{42}$ ya que ambos explican la salud cuando se considera el ambiente psicosocial del trabajo, e identifican el control sobre los contenidos y las condiciones de trabajo, las exigencias psicológicas, el apoyo social, la escasez de recompensas obtenidas a cambio del esfuerzo invertido. Donde el riesgo psicosocial se entiende como el desajuste percibido por el trabajador entre los aspectos mencionados anteriormente. ${ }^{15}$ Lo que explicaría los bajos niveles de satisfacción laboral encontrados sobretodo en los niveles más bajos de la muestra.

Una limitación del estudio es la distribución por sexos, estando casi en su totalidad conformada por hombres, representación que es habitual en minería, y que sería interesante de abordar en otro estudio. Además, debido a lo extenso del instrumento SUSESO-ISTAS 21 por su alto número de subdimensiones (20), para este estudio se optó por analizar sólo las 5 dimensiones.

Se sugiere para futuras investigaciones, abordar el análisis de las subdimensiones, correlacionar con otros indicadores de salud ocupacional, como salud mental, y con indicadores de seguridad laboral, como tasa de accidentalibilidad $u$ otros.

Se recomienda además la posibilidad de utilizar metodología mixta, agregando levantamientos de información de tipo cualitativo, de esta forma, articulando ambas perspectivas, a fin de lograr un análisis más profundo y detenido de los datos observados. Por último, sería interesante comparar los resultados con otras empresas relacionadas al área de la minería e indagar, por ejemplo, similitudes y diferencias en contextos de extracción a rajo abierto y subterráneo.

\section{REFERENCIAS}

1. Peiró $\mathbf{J M}$, Bravo MJ. Factores psicosociales en la prevención de riesgos laborales: oportunidades y retos para la Psicología del Trabajo y de las Organizaciones. Rev Psicol Trab Organ. 1999; 15(2):137-146.

2. Moreira JM, Alvarez MC. Clima organizacional y estrés en una unidad de alto riesgo.Emergencias. 2002;14: 6-12.
3. Salanova M. Organizaciones saludables, organizaciones resilientes. Gestión Práctica de Riesgos Laborales. 2009; 58:18-23.

4. Peiró JM. El Sistema de Trabajo y sus Implicaciones para la Prevención de los Riesgos Psicosociales en el Trabajo. Univ Psychol. 2004; 3(2):179-186. 
5. Martín M, Vera J, Cano M, Molina C. Nuevos retos de las políticas de salud laboral en las organizaciones de trabajo: una aproximación al estrés laboral y al burnout en clave psicosocial. Revista Temas laborales. 2004; 75:187-211.

6. Meliá JL, Nogareda C, Lahera M, Duro A, Peiró JM, Pou R, Salanova, Gracia D, de Bona JM, Bajo JC, Martínez-Losa F. Principios comunes para la evaluación de riesgos psicosociales en la empresa. Perspectivas de Intervención en Riesgos Psicosociales; Evaluación de Riesgos. Barcelona: Foment del Treball Nacional; 2006. p.13-36.

7. Levi L. Factores psicosociales y de organización. En: Sauter L, Murphy L, Hurrel J, Levi L, editores. Enciclopedia de la salud en el trabajo. Vol. 3. Madrid: Ministerio de trabajo y asuntos sociales; 1998.

8. Cuenca R. Introducción a los riesgos laborales de naturaleza psicosocial. Madrid: Instituto Nacional de Seguridad e Higiene en el Trabajo; 1996.

9. Grupo de Trabajo de la comisión sobre Factores de Riesgo Psicosocial en el Trabajo del INSL. Procedimiento general de Evaluación de Riesgos Psicosociales. Pamplona: Fondo de Publicaciones del Gobierno de Navarra; 2005.

10. Romero A. Control y gestión de los riesgos psicosociales. Gestión práctica de riesgos laborales [en línea] 2005 [consultado 15 mar 2009]; 16:1. Disponible en: http://www.graduados-sociales.com/ArticulosCO/AC0194_3.pdf

11. Salanova M, Grau R, Martínez I. Demandas Laborales y Conductas de Afrontamiento: El Rol Modulador de la Autoeficacia Profesional. Psicothema. 2005; 17:390-395.

12. Serra J. Los factores psicosociales como nuevos riesgos emergentes en el contexto del mundo globalizado[en línea] 2005 [consultado 27 mar 2010]. Disponible en:http://www.asepeyo.es/apr/apr0301.nsf/ficheros/PSI0510026\% 20Factores $\% 20$ psicosociales $\% 20$ como $\% 20$ nuevos $\% 20$ riesgos $\% 20$ emergentes\%20en\%20el\%20mundo\%20globalizado.pdf/\$file/PSI0510026\%20 Factores $\% 20$ psicosociales $\% 20$ como $\% 20$ nuevos $\% 20$ riesgos $\% 20$ emergentes\%20en\%20el\%20mundo\%20globalizado.pdf

13. Aranda $C$, Pando $M$, Salazar J, Torres $T$, Alderete $M$, Pérez M. Factores psicosociales laborales y síndrome de burnout en médicos del primer nivel de atención. Investigación en Salud. 2004;6:28-34.

14. Villalobos G. Vigilancia epidemiológica de los factores psicosociales. Aproximación conceptual y valorativa. Cienc Trab. 2004;6:197-201.

15. Moncada $S$, Llorens $C$, Navarro A, Kristensen T. ISTAS 21: Versión en lengua castellana del cuestionario psicosocial de Copenhague Arch Prev Riesgos Labor[en línea] 2005 [consultado 10 nov 2009];8(1):19-21. Disponible en: http://www.scsmt.cat/pdf/8n1orig1.pdf.

16. Moreno N, Llorens $C$, Moncada S. Evaluar e intervenir sobre los riesgos psicosociales: es necesario y posible. La metodología ISTAS21 (CoPsoO). Barcelona: Comisión Obrera Nacional de Catalunya; 2000.

17. Fumagalli A. Bioeconomía y capitalismo cognitivo. Madrid: Traficantes de sueños; 2010.

18. Hernández, D, Salazar, Gómez, V. Relación entre los aspectos psicosociales del ambiente de trabajo y el riesgo cardiovascular en hombres. Revista Latinoamericana de Psicología. 2004;36:107-123.

19. Saavedra N, Fuentealba C, Perez J. Cuestionario de evaluación de Riesgos Psicosociales en el Trabajo. Santiago: Superintendencia de Seguridad Social; 2009

20. Juárez A. Factores psicosociales, estrés y salud en distintas ocupaciones: un estudio exploratorio. Investigación en salud.2007;9(1):56-64.

21. Ansoleaga J. Factores psicosociales laborales asociados a riesgo de sintomatología depresiva en trabajadores de una empresa minera. Salud de los Trabajadores 2010;18:7-16.

22. Vera A, Sepúlveda R, Contreras $G$. Autorreporte de sintomas físicos y correlatos psicosociales en trabajadores de la minería. Cienc Trab. 2006;8:74-78.

23. Sánchez $\mathrm{S}$. Diseño de un modelo causal de satisfacción laboral: aplicación en el sector servicios; [tesis de doctorado]. Córdoba: Universidad de Córdoba; 2006.

24. Locke E. The nature and causes of job satisfaction. En: Dunnette MD, editors. Handbook of Industrial and Organizational Psychology. Chicago, Rand McNally; 1976.

25. Bravo MJ, Peiró JM, Rodríguez I. Satisfacción laboral. En: Peiró J, Prieto $F$, editores. Tratado de psicología del trabajo, Vol. 1. La actividad laboral en su contexto. Madrid: Sintesis S.A.; 1996.p. 343-394.
26. Sánchez-Anguita A. Salud Laboral. Autoeficacia, Ansiedad y Satisfacción. Salamanca: Amarú Ediciones; 2006.

27. IBSON J et al. Organizaciones, conducta, estructura y proceso. México: Interamericana, 1985.

28. Pérez J. NTP 394: Satisfacción laboral: escala general de satisfacción [en línea]. Madrid: INSHT; 2006 [consultado 31 oct 2010]. Disponible en: www.insht.es/ InshtWeb/Contenidos/.../NTP/.../ntp_394.pdf.

29. Caballero K. El concepto de "satisfacción en el trabajo" y su proyección en la enseñanza. Profesorado. 2002;6:1-10.

30. Ruzafa M, Madrigal M, Valendrino A, López L. Satisfacción laboral de los profesionales de enfermería españoles que trabajan en hospitales ingleses. Gaceta Sanitaria. 2008; 22:434-442.

31. Fernández B, Paravic T. Nivel de satisfacción laboral en enfermeras de hospitales públicos y privados de la provincia de Concepción, Chile. Ciencia y Enfermería 2003;2:57-66.

32. Meliá JL, Peiró JM. La medida de la satisfacción laboral en contextos organizacionales: El Cuestionario de Satisfacción S20/23. [The measurement of job satisfaction in organizational settings: The S20/23 Job Satisfaction Questionnaire]. Psicologemas.1989; 5:59-74.

33. Chiang $M$, Salazar $C$, Núñez A. Clima organizacional y satisfacción laboral en un establecimiento de salud estatal: hospital tipo 1. Universidad del Bío-Bío, Chillán. Theoria. 2007;16: 61-76.

34. Machuca $P$, Vera A. Cultura organizacional, estilos de dirección. Trabajo en equipo, contenido del puesto de trabajo, satisfacción laboral y factores psicosociales: un estudio empírico de la calidad de vida laboral en una empresa financiera. [Tesis de magister de administración]. Santiago: Universidad Diego Portales; 2001.

35. García J, Berríos M. El Significado del Trabajo en Personas con Patrón de Conducta Tipo A. Psicothema. 1999; 11(2):357-366.

36. Chile. SUSESO. Cuestionario para diagnosticar la "salud psíquica" de la empresa [en linea] Santiago: SUSESO; 2009 [consultado 10 nov 2009]. Disponible en http://www.suseso.cl/OpenNews/asp/pagDefault.asp?arglnstanciald=1\&argNo ticiald $=197 \&$ argEdicionld $=49$

37. Cano A. Factores psicosociales que inciden en el estrés laboral [en línea]. Madrid: SEAS; 2002 [consultado 10 ene 2011]. Disponible en: http://webcache. googleusercontent.com/search?q=cache:zu9I0MhSml4J:www.ucm.es/info/ seas/estres_lab/fact_psicosoc.htm+saisfaccion+laboral+y+factores+psicosocia les+mineros $\& c d=3 \& h l=e s \& c t=c l n k$.

38. El estrés afecta más a mineros, albañiles y policias [en línea]; Eroski Consumer. Vizcaya:Fundación Eroski; 2008 [consultado 19 ene 2011]. Disponible en http:// www.consumer.es/web/es/salud/2001/07/15/44605.php.

39. Pérez J. El método de evaluación e intervención psicosocial SUSESO ISTAS 21. Santiago: Emerge Asesores; 2010.

40. KARASEK R. Job demands, job decision latitude, and mental strain: Implications for job redesign. Admin Sci Quart. 1979; 24:285-309.

41. KARASEK R, THEORELL T. Healthy work. Stress, productivity, and the reconstruction of working life. New York: Basic Books; 1990.

42. Siegrist, J. Adverse health effects of high-effort/low-reward conditions. J Occup Health Psychol. 1996; 1(1):27-41.

\section{Referencias Complementarias}

Anaya D, Suárez J. Satisfacción laboral de los profesores de educación infantil, primaria y secundaria: un estudio de ámbito nacional. Revista de Educación (Madrid) 2007;(344):217-243.

Organización Internacional del Trabajo (OIT). Enciclopedia de Salud y Seguridad en el Trabajo. Vol. 4. Madrid: Ministerio del Trabajo y Asuntos Sociales OSHA; 1998.

Peiró JM. Desencadenantes del estrés Laboral. Madrid: Edics. Pirámide; 2000.

UGT. Observatorio permanente de riesgos psicosociales. Guía sobre factores y riesgos psicosociales. Madrid: Comisión Ejecutiva Confederal de UGT; 2006.

Vega S. NTP 603: Riesgo psicosocial: el modelo demanda-control-apoyo social (I) [en línea]. Madrid: INSHT; 2003 [consultado 25 sep 2010]. Disponible en: http:// www.insht.es/InshtWeb/Contenidos/Documentacion/FichasTecnicas/NTP/ Ficheros/601a700/ntp_603.pdf. 